\title{
EL CONSENTIMIENTO SEXUAL \\ DE LOS MENORES DE EDAD EN ESPAÑA: \\ CONSIDERACIONES CLÍNICAS \\ Y JURISPRUDENCIALES
}

\author{
José Manuel Bertolín-Guillén \\ Doctor y Licenciado en Medicina y Cirugía; \\ Médico Especialista en Psiquiatría; Licenciado en Psicología \\ E-mail: jmbertolin@,comv.es
}

\begin{abstract}
RESUMEN: Realizamos un análisis crítico, descriptivo y narrativo de la literatura científica psicopatológica internacional y la jurisprudencial española acerca de la sexualidad en menores de edad. Se comentarán, entre abundantes referencias de sanidad y otros preceptos jurídicos, las Leyes Orgánicas 5/2000, Reguladora de la Responsabilidad Penal de los Menores; 1/2004, de Medidas de Protección Integral contra la Violencia de Género; y 1/2015, por la que se modifica la Ley Orgánica 10/1995, del Código Penal. La cuestión del consentimiento sexual de los menores en España y en otros países es delicada y controvertida desde las perspectivas éticas y del amplio ámbito sanitario-jurídico.
\end{abstract}

Palabras clave: Competencia mental; conducta sexual; jurisprudencia; menor de edad; España.

\begin{abstract}
We conduct a descriptive, narrative, critical analysis of the international scientific literature and the national precedent in the case-law on sexuality with minors. The Spanish Organic Laws 5/2000 Regulating Minors' Criminal Liability, 1/2004 about Measures for Comprehensive Protection against Gender Violence, 1/2015 modifying the Spanish Organic Law 10/1995 of the Penal Code will be discussed among other abundant health references and legal precepts. The matter of sexual consent of minors in Spain, just like in other countries, is delicate and quite controversial from the legal, ethical and psychopathological perspective.
\end{abstract}

Keywords: Mental competency; sexual behavior; jurisprudence; minor; Spain.

Sumario: I. Introducción. II. Material Y MÉtodo. III. Resultados: 3.1. Consentimiento Sexual O InCAPACIDAd PARA OPONERSE; 3.2. LA «ALIENACIÓN PARENTAL》; 3.3. EXPERIENCIAS SEXUALES Y PotenCIAL ReSPUESTA Judicial; 3.4. "Disforia De GÉNero» EN LA Adolescencia; 3.5. Rol De La Sanidad EN La SeXualidad De Los MENores; IV. ConCLUSIONES. V. BibLIOGRAFía. 


\section{INTRODUCCIÓN}

La sexualidad es una dimensión natural del ser humano y la adolescencia es una edad crucial para la «salud social». El desarrollo de la sexualidad está directamente vinculado a la dignidad de la persona y al libre desarrollo de la personalidad, como subraya la Ley Orgánica 2/2010, de Salud Sexual y Reproductiva y de la Interrupción Voluntaria del Embarazo. La comunidad médico-pediátrica determina una serie de comportamientos sexuales normales y comunes en los niños. Por otro lado, de modo general son bastante corrientes el abuso, maltrato y negligencia de diversos tipos de los adultos para con niños y adolescentes ${ }^{1}$.

La World Health Organization (OMS, por sus siglas en español) enfatiza que esas conductas inadecuadas, incluyendo las sexuales, tienen como resultado un daño real o potencial a la salud, el desarrollo o la dignidad del niño. Se puede consultar al respecto el Technical Report: WHO Guidelines for the Health Sector Response to Child Maltreat$m e n t^{2}$, de 2019. Por ello, en particular es importante observar que los comportamientos sexuales de los menores de edad sean apropiados de acuerdo a su desarrollo psicobiológico.

Seguidamente abordaremos el estado actual del consentimiento sexual de los menores de edad en España, tal como indica el título de este trabajo, con especial atención en el enfoque de la salud y también de la óptica jurídica. Examinaremos las vigentes nociones del consentimiento sexual del menor (o de su capacidad para oponerse), el controvertido concepto clínico de «alienación parental», la respuesta judicial a las distintas conductas sexuales ilícitas, el reconocido desde hace años como trastorno mental y del comportamiento denominado «Disforia de Género»y, para terminar, el rol que desempeña la sanidad en la sexualidad de los menores.

\section{MATERIAL Y MÉTODO}

Análisis crítico, descriptivo y narrativo de la literatura científica internacional y, en distinta medida, también de la jurisprudencial española, seleccionadas por su calidad y relevancia sobre la capacidad de consentir en niños y adolescentes. Estudiaremos la sexualidad de los menores, con énfasis en las perspectivas sanitario-jurídica y ética.

\section{RESULTADOS}

\subsection{Consentimiento sexual o incapacidad para oponerse}

El Código Penal español vigente fue aprobado por la Ley Orgánica 10/1995. Este código ha sufrido muchas modificaciones a lo largo de los años, la última en 2019. Pero la última de sus grandes reformas se produjo en 2015, con la que se eliminó la diferenciación entre delitos y faltas, pasando las últimas a denominarse delitos leves (art. 33.4). Aunque varían

1 RIZVI, M. B., CONNERS, G. P. and RABINER, J. "New York State child abuse, maltreatment, and neglect". In: StatPearls [Internet]. Treasure Island (FL): StatPearls Publishing; 2021.

2 https://www.who.int/publications/i/item/who-guidelines-for-the-health-sector-response-to-child-maltreatment 
las diferentes legislaciones homónimas de Europa, suelen existir puntos comunes, cosa que no sucede igual con otros lugares y culturas distintas.

En algún estudio reciente se ha comprobado que la exposición a los medios de comunicación social sexualmente explícitos en la adolescencia temprana ha tenido una relación sustancial con el comportamiento sexual de riesgo en la adultez emergente ${ }^{3}$. Asimismo, es un hecho que asistimos a la erotización anticipada del cuerpo de las niñas en el llamado «período de latencia» ${ }^{4}$, manifestado en las formas hipersexualizadas que en ocasiones asume la moda infantil ${ }^{5}$. En general, para muchos autores la conducta hipersexualizada por debajo de los quince años de edad suele considerarse como temprana o prematura. Como contrapunto, la familia y la escuela suelen comportarse como entornos «erotofóbicos» ${ }^{6}$.

Mención específica y por separado merece el turismo sexual infantil, principalmente por parte de hombres adultos ${ }^{7}$. Y en particular para la medicina psiquiátrica forense es muy relevante el delito sexual infantil. Es sabido que la pedofilia en concreto es un importante factor de riesgo en la reincidencia del delincuente sexual. Pero el concepto mismo de abuso sexual infantil es difícil de definir y son considerables los problemas éticos ligados a la recopilación de datos al respecto ${ }^{8}$. En la práctica es frecuente entre adolescentes dicotomizar entre una relación satisfactoria, que significará para ellos que ha sido consentida, y la no satisfactoria que implicará que ha sido coercitiva9 ${ }^{9}$. En el adecuado consentimiento del menor habrá que tener muy en cuenta los poderes institucionales e interactivos que concurran como condicionantes o determinantes de dicho asentimiento.

Según un estudio alemán sobre 1194 personas, de las que 564 eran mujeres, los grados de gravedad de síntomas de hipersexualidad en adultos se asociaban a menudo con graves dificultades intra e interpersonales ${ }^{10}$. Desde el punto de vista de la salud mental la hipersexualidad puede ser evaluada por cuestionarios como el Hypersexual Disorder Screening Inventory y el Hypersexual Behavioral Consequences Scale, que se suelen utilizar en entornos clínicos para medir su gravedad y trazar un mapa de áreas potenciales de deterioro personal del sujeto.

3 LIN, W. H., LIU, C. H. and YI, C. C. "Exposure to sexually explicit media in early adolescence is related to risky sexual behavior in emerging adulthood". PLoS One, 2020; vol. 15 (núm. 4), pp. e0230242.

4 Usamos aquí el concepto de la clásica terminología Freudiana que subraya la etapa del crecimiento que permite al individuo desarrollar una estructura de personalidad más compleja.

5 DÍAZ-BUSTAMANTE-VENTISCA， M., LlOVET-RODRÍGUEZ，C. y NARROS-GONZÁLEZ， M. J. «Sexualización percibida en los estilismos de moda de niñas: un análisis transcultural en España-China». Comunicar, 2020; vol. XXVIII (núm. 65), pp. 77-87.

6 RODRÍGUEZ-MOLINERO, L. «La adolescencia y los riesgos sexuales» [Editorial]. Adolescere, 2019; vol. 7 (núm. 1), pp. 3-4.

7 KOOPS, T., TURNER, D., NEUTZE, J. and BRIKEN, P. "Child sex tourism - prevalence of and risk factors for its use in a German community sample". BMC Public Health, 2017; vol. 17 (núm. 1), pp. 344.

8 BERTOLÍN-GUILLÉN, J. M. Deontología y confidencialidad en psiquiatría y psicología clínica en España. Revista de Bioética y Derecho, 2021; [vol. 23] (núm. 52), pp. 173-83.

9 COHEN, N. L. and ŠTULHOFER, A. "Correlates of adolescent ambiguity in defining their experience of sexual victimization in two large-scale Croatian samples". Journal of Child \& Adolescent Trauma, 2018; vol. 12 (núm. 2), pp. 211-20.

10 ENGEL, J., KESSLER, A., VEIT, M., SINKE, C., HEITLAND, I., KNEER, J. et al. "Hypersexual behavior in a large online sample: Individual characteristics and signs of coercive sexual behavior". Journal of Behavioral Addictions, 2019; vol. 8 (núm. 2), pp. 213-22. 
Los países de la Unión Europea fijan entre los catorce y dieciocho años la edad de consentimiento para ambos sexos. En nuestro país la edad del consentimiento sexual o de la capacidad para oponerse era antes de las más bajas de la Unión. Actualmente y desde 2015, todo el que realizare actos sexuales con un menor de dieciséis años será castigado con pena de prisión como responsable de abuso sexual (art. 183 del Código Penal), pues con la reforma de 2015 se elevó la edad del consentimiento. En circunstancias de engaño, abuso de autoridad, de confianza o de influencia sobre la víctima también se cometerá delito sexual si la persona damnificada es mayor de dieciséis y menor de dieciocho años.

Los menores de edad no emancipados no pueden realizar cualquier acto con la debida capacidad de obrar. Ese defecto de la capacidad ha de suplirse con la patria potestad o la tutela. Así pues, en ellos se dan o pueden darse límites de la autonomía de la voluntad contractual para consentir relaciones sexuales. Tras la reforma de 2015, nuestro Código Penal estableció una presunción iuris tantum (como es bien sabido, este latinismo significa presunción del hecho, pero que permite la prueba en contrario, a diferencia de la iuris et de iure) de falta de capacidad de los menores de dieciséis años para consentir relaciones sexuales. Es excepción si se tratara de relaciones aceptadas con una persona de edad, grado de desarrollo físico y madurez mental próximos al menor. Así pues, no es cuestión solo de la presunta fragilidad o vulnerabilidad de la posible víctima. En todo caso, el perdón del ofendido no extingue la acción ni las responsabilidades penales.

Véase también, entre otros, el art. 189 del capítulo V del Código Penal, que contempla algunos tipos de delito contra la libertad e indemnidad sexuales, aunque una minoría de autores los entiende como un bien jurídico innecesario. También puede verse al respecto y de modo principal la Doctrina de la Fiscalía General del Estado, Circular 1/201711, sobre la interpretación del art. 183.4 del citado código. Igualmente, conviene tener presentes las leyes orgánicas 5/2000, Reguladora de la Responsabilidad Penal de los Menores, con última modificación de 2012, así como la 5/2010 que modificó el Código Penal de 1995 e incorporó en el Título VIII del Libro II, el Capítulo II bis denominado: «De los Abusos y Agresiones Sexuales a Menores de Trece Años».

El Congreso de los Diputados aprobó en abril de 2021 el novedoso Proyecto de Ley Orgánica de Protección Integral a la Infancia y la Adolescencia Frente a la Violencia, que se había presentado el año anterior y que sitúa a nuestro país como vanguardista en la protección de los derechos de los menores. El proyecto contempla la posible, aunque también discutida, creación de juzgados específicos y de otras entidades. En la actualidad se protege penalmente en España de forma limitada a los menores que se hallen entre los dieciséis y diecisiete años. A partir de los dieciséis está despenalizado el sexo consentido entre un adulto y un menor.

La Ley española establece la protección de la infancia y la adolescencia frente a la violencia o para salvaguardar como fin primordial el interés superior de las personas menores de edad. El interés superior del menor es un concepto de difícil concreción, que tiene una triple dimensión en tanto que es derecho, principio y norma de procedimiento. Hay autores $^{\mathbf{1 2}}$ que subrayan la importancia combinada de las características del sujeto menor de edad, de su entorno y de la predictibilidad para alcanzar su mejor desarrollo integral.

11 https://www.boe.es/buscar/abrir_fiscalia.php?id=FIS-C-2017-00001.pdf

12 LÓPEZ-CONTRERAS, R. E. «Interés superior de los niños y niñas: definición y contenido». Revista Latinoamericana de Ciencias Sociales, Niñez y Juventud, 2015; vol. 13 (núm. 1), pp. 51-70. 
Será necesario, por tanto, establecer lo más objetivamente que sea posible lo que convenga más al menor para el óptimo desarrollo de su personalidad. Con relación a los abusos y agresiones sexuales a menores en nuestro país, se puede consultar a García $(2020 \text {, p. 18) })^{13}$ Respecto a las correspondientes periciales forenses médicas o psicológicas ante los tribunales de justicia será muy trascendente la idoneidad del perito basada en su formación, óptima capacitación o cualificación profesional titulada y experiencia, dada la realidad de que se producen en ocasiones errores clínicos y falsas denuncias de abuso sexual infanti1 ${ }^{14,15,16}$.

Hay que señalar que el Consejo General del Poder Judicial aprobó en febrero de 2021 el informe sobre el anteproyecto de la Ley Orgánica de Garantía Integral de la Libertad Sexual. Pero, a su vez, dicho informe fue criticado en marzo en el documento de Propuestas de Enmiendas al proyecto de Ley, un dosier de 63 págs. de la asociación $\mathrm{Mu}-$ jeres Juezas de España y que está dirigido a los grupos parlamentarios ${ }^{17}$. Como se puede constatar, son numerosas las propuestas de lege ferenda (usando la también muy habitual expresión latina común en la literatura jurídica que enfatiza la necesidad de cambios) planteadas como alternativas a la correspondiente regulación positiva actual ${ }^{18}$.

\subsection{La «alienación parental»}

Es relevante señalar la propuesta que se ha incluido en el referido Proyecto de Ley Orgánica de Protección Integral..., de abril de 2021 y mencionado en el apartado anterior, de prohibir en España el llamado popularmente Síndrome de Alienación Parental o, más simplemente, alienación parental. Aunque no todos están de acuerdo, el síndrome ha sido considerado seudocientífico y se ha excluido de los sistemas taxonómicos Diagnostic and Statistical Manual of Mental Disorders, de la American Psychiatric Association -APA(DSM-5) de 2013 y del próximo International Statistical Classification of Diseases and Related Health Problems (ICD-11, o CIE-11 por sus siglas en español), de la OMS, que tiene previsto entrar en vigor mundial en 2022. La alienación parental es considerada un problema de relación padres-hijos o de ambos padres, pero los problemas de relación per se no se consideran trastornos mentales.

Ahora bien, hay países como la República Federativa de Brasil en que la alienación parental es un término legal que requiere de un estudio pormenorizado para cada hipotético caso $^{19}$. La supuesta alienación parental debe distinguirse del alejamiento o re-

13 GARCÍA-FERNÁNDEZ, M. A. «Delitos sexuales contra menores: especial referencia a agresiones y abusos sexuales». Revista Internacional de Doctrina y Jurisprudencia, 2020; vol. 23, pp. 15-43.

14 KVITKO, L. A. «Errores periciales y falsas denuncias de abuso sexual infantil». Revista Española de Medicina Legal, 2006; vol. 6, pp. 39-42.

15 TORRES-FERNÁNDEZ, M. E. «Abusos sexuales sobre personas incapacitadas para oponerse a su agresor y sobre incapaces para consentir». Revista Española de Medicina Legal, 2020; vol. 46 (núm. 4), pp. 198-9. [Article in Press]

16 BERTOLÍN-GUILLÉN, J. M. «Las especialidades facultativas del ámbito de la salud mental ante los tribunales de justicia». Gaceta Internacional de Ciencias Forenses, 2021. [Article in Press]

17 http://www.mujeresjuezas.es/wp-content/uploads/2020/09/PROPUESTAS-LOIA-AMJE.pdf

18 SANCHO-CONDE, T. Minoría de edad y delincuencia sexual: consecuencias jurídicas aplicables a menores que cometen delitos contra la libertad e indemnidad sexual. [Tesis doctoral]. Tarragona: Departamento de Derecho Público, Universitat Rovira i Virgili; 2020.

19 PENA, R., LAUAR, H. and BARROS, A. "Forensic psychiatry assessment during parental alienation claims: Two cases with different outcomes". Brazilian Journal of Psychiatry, 2020; vol. 42 (núm. 4), pp. 453-4. 
chazo justificado hacia uno de los padres y se caracteriza típicamente por la presencia de una campaña de denigración hacia un progenitor previamente querido por el menor. Dicha campaña se inicia instigando temor y animadversión injustificadas y suele producirse durante el litigio por la custodia en un proceso judicial de divorcio ${ }^{20,21,22,23,24}$.

A pesar de que se niega su entidad clínica, la exposición infantil a la llamada alienación parental se ha relacionado con la mayor probabilidad de sufrir síntomas depresivos y una disminución de los resultados en la edad adulta de la escala Health-Related Quality of Life ${ }^{25}$ Recientemente se ha sugerido, además, una escala de manipulación judicial para medir la disposición a mentir y a utilizar a los niños para dañar al otro padre, y que se podría emplear en la práctica profesional ${ }^{26}$.

Algunos autores han denominado «divorcio patológico» al que resulta altamente conflictivo, aunque los implicados estén exentos de patologías mentales ciertas o en desarrollo. Precisamente en tales casos es frecuente la supuesta alienación parental. Esta implica las manifestaciones psicopatológicas observadas en niños que se hallan sujetos a separaciones parentales altamente conflictivas ${ }^{27}$. Dicha psicopatología de los menores puede tener, según casos, consecuencias trágicas y constituir incluso un trastorno o condición mental patológica grave ${ }^{28}$.

\subsection{Experiencias sexuales y potencial respuesta judicial}

En España la educación sexual actual de los menores resulta casi anecdótica, a pesar de que está publicado desde 2011 un documento de recomendaciones de consenso al respecto ${ }^{29}$. El 20\% de los adolescentes entre quince y dieciséis años reconoce haber mantenido relaciones sexuales coitales, según el Health Behaviour in Schoolaged Children, que es

20 MAIDA, A. M., HERSKOVIC, V. y PRADO, B. «Síndrome de alienación parental». Revista Chilena de Pediatría, 2011; vol. 82 (núm. 6), pp. 485-92.

21 VILALTA, R. y NODAL, M. W. «Sobre el mito del síndrome de alienación parental (SAP) y el DSM-5». Papeles del Psicólogo, 2017; vol. 38 (núm. 3), pp. 224-31.

22 MOON, D. S., LEE, M. H., CHUNG, D. S. and KWACK, Y. S. "Custody evaluation in high-conflict situations focused on domestic violence and parental alienation syndrome". Soa Chongsonyon Chongsin Uihak, 2020; vol. 31 (núm. 2), pp. 66-73.

23 VERROCCHIO, M. C., MARCHETTI, D., CARROZZINO, D., COMPARE, A. and FULCHERI, M. "Depression and quality of life in adults perceiving exposure to parental alienation behaviors". Health and Quality of Life Outcomes, 2019; vol. 17 (núm. 1), pp. 14.

24 CLEMENTE, M., PADILlA-RACERO, D. and ESPINOSA, P. "The dark triad and the detection of parental judicial manipulators. Development of a judicial manipulation scale". International Journal of Environmental Research, 2020; vol.17 (núm. 8), pp. 2843.

25 BENSUSSAN, P. «Aliénation parentale, abus psychologique de l'enfant et DSM-5». L'Encéphale, 2017; vol. 43 (núm. 6), pp. 507-9.

26 VILJOEN, B. L. "Unintentional state enforced parental alienation syndrome during the hard lockdown in South Africa". The Family Journal, 2021. [OnlineFirst]

27 HURTADO-MURILlO, F. y PÉREZ-CONCHILLO, M., coord. Educación para la sexualidad con bases cientificas. Documento de consenso de Madrid. Recomendaciones de un grupo internacional de expertos. Valencia, ES: Asociación Española de Especialistas en Sexología; 2011.

28 MORENO, C., RAMOS, P., RIVERA, F., SÁNCHEZ-QUEIJA, I., JIMÉNEZ-IGLESIAS, A., GARCÍA-MOYA, I. et al. Resultados del estudio HBSC 2018 en España sobre conducta sexual. Análisis de tendencias 2002-20062010-2014-2018. Madrid: Ministerio de Sanidad; 2020.

29 HRUZ, P. W. "Deficiencies in scientific evidence for medical management of gender dysphoria". The Linacre Quarterly, 2020; vol. 87 (núm. 1), pp. 34-42. 
un importante estudio internacional auspiciado por la OMS y en el que participan casi cincuenta países occidentales.

Del grupo de adolescentes mayores de quince años que ha experimentado relaciones coitales, el 10,3\% las mantuvieron por primera vez a los trece años de edad o antes, siendo los porcentajes mayores en chicos $(12,4 \%)$ que en chicas $(8,0 \%)^{30}$. El porcentaje de adolescentes con prácticas de riesgo en 2002 era el 16,1\%, que se elevó en 2018 al 24,6\%. En relación específica con la explotación y abuso sexuales, puede consultarse de forma muy genérica la Convention on the Rights of the Child ${ }^{31}$, que es un tratado internacional adoptado por la Asamblea General de la ONU en 1989, en vigor desde el año siguiente, y en particular los art. 34 y otros.

El asunto de la edad para consentir continúa siendo en la actualidad bastante polémico. Al respecto se puede seguir en los medios de comunicación social el reciente caso absolutorio de abril de 2021 de la Audiencia Provincial de Navarra (España) de un delito de abuso sexual a una menor de catorce años (n. ${ }^{\circ}$ de Resolución: 81/2021) ${ }^{32}$. El fallo del tribunal probablemente será recurrido por la Fiscalía de lo penal. La chica era hijastra del acusado, quedó embarazada y dio a luz a una niña. La menor exculpó al padrastro para quien se solicitaban seis años de prisión.

Esa sentencia es coincidente en el tiempo con el nuevo Proyecto de Ley Orgánica de Protección Integral..., de abril de 2021, al que hemos aludido aquí varias veces. En otro asunto de 2016 sobre un delito continuado de abuso sexual con prevalimiento, la Fiscalía recurrió la sentencia dictada por la Sala de lo Civil y Penal del Tribunal Superior de Justicia de Navarra sobre la popularmente llamada «la manada». Ha de tenerse en cuenta que la declaración de la víctima puede ser considerada prueba de cargo suficiente para enervar la presunción de inocencia, según dicta la Sala de lo Penal del Tribunal Supremo en la sentencia n. $.^{\circ} 257 / 2021$, de 24 de febrero.

Puede verse también, entre otras muchas, la sentencia n. ${ }^{0}$ 127/2014 de la Sección 4. ${ }^{a}$ de la Audiencia Provincial de Valladolid, de 19 de marzo. De igual modo, para toda España conviene conocer el documento titulado: «La Respuesta Judicial a la Violencia Sexual que Sufren los Niños y las Niñas» ${ }^{33}$, de la Delegación del Gobierno Contra la Violencia de Género, del Ministerio de Igualdad, de 2020.

\section{4. «Disforia de género» en la adolescencia}

La actual clasificación internacional de las enfermedades ICD o CIE-10 (OMS, 1992), aunque no reconoce específicamente la denominación concreta de «Disforia de Género», aborda el equivalente llamado en esa taxonomía: «Trastorno de la Identidad Sexual en la Infancia» (código F64.2). La CIE-10 enfatiza que dicho trastorno no se tiene que confundir con la falta de conformidad con el rol sexual socialmente aceptado, que resulta mucho más frecuente pero que no es un trastorno. Ni se debe confundir tampoco con otras realidades que sí son trastornos mentales en la CIE-10, como el «Transvestismo No Fetichista» (F64.1) o el «Transvestismo Fetichista» (F65.1).

30 MORANT-LUJÁN, Y. «Intersexualidad. ¿Qué es normal?» Revista Desexología, 2020; vol. 9 (núm. 1), pp. 4-6.

$31 \mathrm{https} / / /$ www.refworld.org/docid/3ae6b38f0.html

32 https://www.poderjudicial.es/search/AN/openDocument/a87ea29782a8bcc4/20210421

33 https://violenciagenero.igualdad.gob.es/violenciaEnCifras/estudios/investigaciones/2020/pdfs/respuestajudicial.pdf 
En la futura y próxima CIE-11, que como hemos dicho está previsto que sea oficial para 2022, ya se contemplará el diagnóstico que se llamará en ella «Incongruencia de Género» entre las denominadas en su capítulo 17: «Condiciones Relacionadas con la Salud Sexual», junto a otros conceptos como son las disfunciones sexuales o los trastornos de dolor sexual. La incongruencia de género sustituirá como diagnóstico al de «Transexualismo» (F64.0, de la aún vigente CIE-10). En cambio, el también vigente DSM-5 de 2013, de la APA, tipifica claramente el diagnóstico de «Disforia de Género», y lo subdivide en niños (código 302.6), en adolescentes y adultos (302.85), y cuando no se puede especificar (codificado también 302.6, que es equivalente a los igualmente vigentes: «Trastornos de la Identidad Sexual Sin Especificación», con código F64.9 de la CIE -10). La disforia de género del DSM-5 hace referencia al malestar psíquico y clínicamente significativo que suele acompañar a la marcada incongruencia de género.

El área del sexo y del género es muy controvertida. Resulta fácilmente observable que está surgiendo una cohorte emergente de variados y supuestos «especialistas» o «expertos» en medicina «transgénero» ${ }^{34}$. Transgénero o coloquialmente «trans», es un término general que se utiliza para describir a las personas con diversidad de género. La polisemia relacionada es considerable. Los cambios en la presentación y rol social de las personas transgénero son típicamente observables. La comunidad científica a menudo se plantea preguntas sobre el papel de las redes sociales en la emergencia y el desarrollo de la disforia de género. Se acepta que pueda haber también la conocida como disforia anatómica sin trastorno del desarrollo sexual.

Una cuestión de importancia creciente en nuestro país, así como en otros muchos, es lo referente a la intersexualidad y transexualidad en tanto que afectan a los menores de edad. Estos asuntos están suficientemente descritos en la moderna literatura médico-psiquiátrica y psicológico-clínica internacional. Tales condiciones o categorías pueden estar biológicamente condicionadas o ser solo pretendidas en función de la identidad de género y sexual del afectado, identidades que pueden o no ser coincidentes ${ }^{35,36,37}$. Además, ha de tenerse en cuenta que, en general, las minorías sexuales y de género están subrepresentadas en los estudios de salud ${ }^{38}$.

Las intervenciones hormonales y quirúrgicas para alcanzar la corporeidad de género deseada, son casi siempre «normales» en adultos. Pero cuando esa elección ocurre en menores, sobre todo cuando hay una identidad de género disconforme con el asignado y se pretende conseguir una nueva identidad sexual, puede tener consecuencias imprevisibles y potencialmente irreversibles. Por ello será tan importante el consentimiento válido, también denominado consentimiento informado o legítimamente manifestado,

34 GÓMEZ-BALAGUER, M., HURTADO-MURILlO, F., MODREGO-PARDO, I. y MORILLAS-ARIÑO, C. «Identidad de género e identidad sexual: a veces coincidentes, pero no siempre lo mismo». Revista Desexología, 2020; vol. 9 (núm. 2), pp. 5-7.

35 BERTOLÍN-GUILLÉN J. M. "Intersexuality, transsexuality and mental health". Annals of Psychiatry \& Mental Health, 2020; vol. 8 (núm. 3), pp. 1156.

36 PATTERSON, J. G., JABSON, J. M. and BOWEN, D. J. "Measuring sexual and gender minority populations in health surveillance". LGBT Health, 2017; vol. 4 (núm. 2), pp. 82-105.

37 MURPHY, T. F. “Adolescents and body modification for gender identity expression”. Medical Law Review, 2019; vol. 27 (núm. 4), pp. 623-39.

38 BERTOLÍN-GUILLÉN, J. M. "Informed consent and mental health, mainly in Spain, EU". International Journal of Psychiatry and Mental Health, 2020; vol. 2 (núm. 1), pp. 1-7. 
del peticionario ${ }^{39,40}$. El marco legal mínimo actual en nuestro país, básico pero obligatorio, además de las leyes autonómicas que correspondan, es la Ley General de Sanidad 14/1986. Esta Ley está sustituida en esta cuestión por la Ley 41/2002, Básica Reguladora de la Autonomía del Paciente y de Derechos y Obligaciones en Materia de Información y Documentación Clínica, a la que completa y actualiza.

Entre 1995 y 2007 destaca la elevada ausencia de alteraciones psicopatológicas en los casos de transexualidad peritados por médicos forenses adscritos a los servicios clínicos médico-forenses de los institutos de medicina legal ${ }^{41}$. Lo habitual, sin embargo, es que los funcionarios titulares médicos forenses de la Administración de Justicia no estén también especializados en psiquiatría por el Estado ${ }^{42}$, por lo que su capacitación en este ámbito tan concreto podría no ser la óptima. Véase el Real Decreto 296/1996, por el que se aprueba el Reglamento Orgánico del Cuerpo de Médicos Forenses, con última actualización de 2005. Puede verse también el Real Decreto 704/2020 que va a regular en adelante la especialidad en medicina legal y forense en España.

Los asuntos vinculados con el constructo social del género personal son de la mayor vigencia mediática y científica en el mundo occidental. Sin embargo, existe igualmente el riesgo de extender indiscriminadamente e incluso fomentar de forma incorrecta, sobre todo en menores, todo lo relacionado con el concepto transgénero, como si se tratara únicamente de mero alejamiento ideológico del pensamiento dicotómico o binario. Se puede partir del hecho de que existen tres grados de diferenciación sexual biológica: el genético, el gonadal y el genital. La mayoría de sujetos menores de edad experimentan congruencia psíquica entre su sexo biológico, identidad de género y rol sexual.

En nuestro país resulta imperativa la Ley 3/2007 Reguladora de la Rectificación Registral de la Mención Relativa al Sexo de las Personas, con última enmienda de 2019, además de las pertinentes leyes autonómicas, como: la LEY 2/2014, de 14 de abril, por la Igualdad de Trato y la No Discriminación de Lesbianas, Gays, Transexuales, Bisexuales e Intersexuales en Galicia; la Ley 3/2016, de 22 de julio, de Protección Integral Contra LGTBIfobia y la Discriminación por Razón de Orientación e Identidad Sexual en la Comunidad de Madrid; la 8/2017, de 7 de abril, Integral del Reconocimiento del Derecho a la Identidad y a la Expresión de Género en la Comunitat Valenciana; y otras varias.

Las comunidades de Madrid, Aragón, Valencia y Andalucía prohíben de forma taxativa las intervenciones terapéuticas o psicoterapias homófobas. Por la antes citada Ley 3/2007 se modificaron en España las leyes sobre el Registro Civil de 1957 y sobre la expedición del Documento Nacional de Identidad de 1978. La discriminación de cualquier tipo está prohibida por el Convenio Europeo para la Protección de los Derechos Humanos y de las Libertades Fundamentales, en vigor desde 1953, y por la versión actualizada en 2007 de la Carta de los Derechos Fundamentales de la Unión Europea.

39 MAROTE-GONZÁLEZ, R. M., PERA-BAJO, F. J., PEREA-PÉREZ, B. y SÁNCHEZ-SÁNCHEZ, J. A. «Estudio de la transexualidad desde la Clínica Médico Forense de Madrid». Revista Española de Medicina Legal, 2018 ; vol. 44 (núm. 4), pp. 150-7.

40 KALTIALA-HEINO, R., BERGMAN, H., TYÖLÄJÄRVI, M. and FRISÉN, L. "Gender dysphoria in adolescence: Current perspectives". Adolescent Health, Medicine and Therapeutics, 2018; vol 9, pp. 31-41.

41 XU, Y., NORTON, S. and RAHMAN, Q. "Adolescent sexual behavior patterns in a British birth cohort: A latent class analysis". Archives of Sexual Behavior, 2021; vol. 50 (núm. 1), pp. 161-80.

42 Ibid. BERTOLÍN-GUILLÉN, J. M. «Las especialidades facultativas del ámbito de la salud mental ante los tribunales de justicia». Gaceta Internacional de Ciencias Forenses, 2021. [Article in Press] 
Parece realista, aunque pueda disgustar a algunas personas y grupos muy polarizados, la posibilidad de que niños prepúberes con conductas de variación de género sean prematura y erróneamente tipificados y tratados como transgéneros. Véase al respecto la peculiar campaña internacional Stop Trans Pathologization. Sea por el motivo que sea, lo cierto es que los adolescentes con marcada disforia de género, con o sin trastorno orgánico del desarrollo sexual y que buscan tratamiento en los servicios de identidad de género, muestran considerable comorbilidad psiquiátrica coincidente ${ }^{43}$.

Por último, se ha detectado una consistencia o coherencia moderada entre la intimidad con personas del mismo sexo y la atracción autodeclarada por el mismo sexo en niños varones, consistencia que es baja para las niñas. En el mismo estudio, las conductas sexuales de baja intensidad, exclusivamente con el otro sexo y sin intimidad entre personas del mismo sexo, representaron el $34,21 \%$, solo por debajo de quienes no tenían ninguna aproximación sexual a otros menores ${ }^{44}$.

\subsection{Rol de la sanidad en la sexualidad de los menores}

Como se comentaba en el apartado anterior, es cada vez más común que las personas transgénero y de género no binario bloqueen su transición a la pubertad o utilicen terapia hormonal para obtener el fenotipo del género deseado. Sin embargo, se sabe poco sobre el impacto de estas manipulaciones en el cerebro ${ }^{45}$. En estudios de 2005 a 2007, para la German Network of Disorders of Sex Development/Intersexuality los trastornos del desarrollo sexual son un grupo heterogéneo de patologías en relación con la determinación del sexo o de la diferenciación sexual, todas ellas consideradas condiciones raras ${ }^{46}$. Es decir, son estados congénitos atípicos de falta de coincidencia del sexo cromosómico, gonadal y fenotípico, probablemente influidos por múltiples factores ${ }^{47}$ Desde 1959 se ha avanzado considerablemente en el conocimiento de las influencias permanentes que tiene la exposición temprana a los andrógenos en el comportamiento posterior de los individuos que recibieron ese tratamiento hormonal ${ }^{48,49}$.

En particular, los facultativos especialistas médicos de la salud pueden intervenir en los casos concretos de las siguientes maneras: Primero, establecer si el sujeto menor de

43 Ibid. KALTIALA-HEINO, R., BERGMAN, H., TYÖLÄJÄRVI, M. and FRISÉN, L. "Gender dysphoria in adolescence: Current perspectives". Adolescent Health, Medicine and Therapeutics, 2018; vol 9, pp. 31-41.

44 XU, Y., NORTON, S. and RAHMAN, Q. "Adolescent sexual behavior patterns in a British birth cohort: A latent class analysis”. Archives of Sexual Behavior, 2021; vol. 50 (núm. 1), pp. 161-80.

45 NGUYEN, H. B., LOUGHEAD, J., LIPNER, E., HANTSOO, L., KORNFIELD, S. L. and EPPERSON, N. "What has sex got to do with it? The role of hormones in the transgender brain". Neuropsychopharmacology, 2019; vol. 44 (núm. 1), pp. 22-37.

46 LUX, A., KROPF, S., KLEINEMEIER, E., JÜRGENSEN, M., THYE, U. and THE DSD NETWORK WORKING GROUP. "Clinical evaluation study of the German network of disorders of sex development (DSD)/ intersexuality: Study design, description of the study population, and data quality". BMC Public Health, 2009; vol. 9, pp. 110.

47 GARCÍA-ACERO, M., MORENO-NIÑO, O., SUÁREZ-OBANDO, F., MOLINA, M., MANOTAS, M. C., PRIETO, J. C. et al. "Disorders of sex development: Genetic characterization of a patient cohort". Molecular Medicine Reports, 2020; vol. 21 (núm.1), pp. 97-106.

48 Phoenix, C. H., Goy, R. W., Gerall, A. A. and Young, W. C. "Organizing action of prenatally administered testosterone propionate on the tissues mediating mating behavior in the female guinea pig". Endocrinology, 1959; vol. 65 (núm. 3), pp. 369-82.

49 HINES, M. "Neuroscience and sex/gender: Looking back and forward". Journal of Neuroscience, 2020; vol. 40 (núm. 1), pp. 37-43. 
edad sufre un trastorno del desarrollo sexual, es decir, si padece una desviación de la normalidad somática o biológica innata, entendida tal normalidad únicamente en el contexto de la capacidad reproductiva. En segundo lugar, determinar si ha manifestado conducta sexual atípica aparecida en su infancia temprana, entendida esa conducta únicamente como diferente de la comúnmente esperada. En tercer lugar, determinar si el individuo manifiesta en el momento actual identificación con la identidad de otro sexo no coincidente con su sexo gonadal y, por tanto, incongruente con su sexo o género manifestado y asignado formalmente en el nacimiento.

De igual modo, desde la perspectiva sanitaria se tendrá que determinar si la persona expresa habitualmente, tanto discursiva como conductualmente, que posee un género sexual atípico (en el mero sentido estadístico) o disconforme con el género esperado. Asimismo, si eso le ocasiona malestar psíquico o insatisfacción cognitivo-afectiva relevantes, es decir, incongruencia de género, considerado como verdadero problema actual identificado y tipificado por un facultativo especialista competente y suficiente como para tener entidad clínica diagnosticable.

También habrá que determinar con perspectiva sanitaria si en la actualidad el sujeto menor de edad desea convertirse en persona transexual mediante tratamiento hormonal y cirugía de reasignación de sexo; es decir, si hay deseo manifestado explícitamente de liberarse de sus originales caracteres sexuales primarios y secundarios. Y, por último, en el específico criterio del facultativo especialista oficial o titulado en salud mental que intervenga, se tendrá que descartar que el-individuo esté sufriendo en la actualidad, según la CIE-10, un Trastorno de la Inclinación Sexual o trastorno parafílico (F65). Acerca de la despatologización de la condición transgénero en la próxima CIE-11 puede consultarse a Robles y Ayuso (2019) ${ }^{\mathbf{5 0}}$.

Todas las cuestiones comentadas hasta aquí son muy considerables, sobre todo si ocurren en menores edad. Ya para terminar, sobra decir que la orientación sexual en sí misma no es un trastorno. Pero ha de tenerse en cuenta que el individuo pudiera experimentar, en particular, una orientación sexual egodistónica (F66.1). Según la CIE-10, el trastorno de la identidad sexual en la infancia (F64.2) puede o no evolucionar a transexualidad (F64.0) en el adulto. Ha de considerase, además, que el cambio genital no garantiza el buen ajuste cognitivo y afectivo al sexo elegido.

\section{CONCLUSIONES}

La cuestión del consentimiento sexual de los menores, tal como ha sido explicitada hasta aquí y como puede constatarse con facilidad, es delicada desde las perspectivas legal, ética y patológica. En particular afecta a la medicina forense en tanto que ésta aplica los conocimientos médicos y biológicos para la resolución de los problemas que plantea el derecho.

Es importante también para los médicos de distintas especialidades diferentes de la pediatría y la psiquiatría y que se consideren expertos en sexología, entendida ésta como una disciplina autónoma. Todos ellos examinarán con preferencia el comportamiento se-

50 ROBLES-GARCÍA R y AYUSO-MATEOS J. L. «CIE-11 y la despatologización de la condición transgénero» [Editorial]. Revista de Psiquiatría y Salud Mental (Barc.), 2019; vol. 12 (núm. 2), pp. 65-7. 
xual y sus diferentes manifestaciones. Ahora bien, como la sexología no es propiamente una especialidad oficial médica ni psicológica es proclive al intrusismo «paracientífico».

Desde las ciencias de la salud mental es relevante el controvertido concepto de la alienación parental. La moderna taxonomía psicopatológica reconoce típicamente el diagnóstico clínico de Disforia de Género o Trastorno de la Identidad Sexual en la Infancia (F64,2, CIE-10 y 302.6, DSM-5) y en adolescentes y adultos (302.85, DSM-5)). De modo general, la sanidad tiene mucho que decir en la sexualidad de los menores, lo que puede contrastar con algunas modas pretenciosas y populistas.

Hemos visto también que las experiencias sexuales de niños y adolescentes en ocasiones requieren de la oportuna respuesta judicial. En julio de 2015 entró en vigor en España la reforma del Código Penal que elevó el consentimiento sexual de los trece a los dieciséis años. Es la misma edad que han fijado otros miembros de la Unión Europea como Bélgica, Luxemburgo, Países Bajos y Noruega. Hasta entonces las relaciones con un menor de trece o más años eran legales en nuestro país si eran consentidas.

Después de 2015 las relaciones sexuales ya eran delito en España si por el menor no se habían cumplido los dieciséis. Hay un margen en que puede haber consentimiento válido, que estará condicionado a la proximidad en edad y madurez de los partícipes. En algún país europeo fuera de la Unión como Suiza, se ha concretado legalmente una diferencia etaria máxima de tres años en los implicados para poder determinar que no haya delito.

\section{BIBLIOGRAFÍA}

Bensussan, P. «Aliénation parentale, abus psychologique de l'enfant et DSM-5». L'Encéphale, 2017; vol. 43 (núm. 6), pp. 507-9. https://doi.org/10.1016/j.encep.2017.11.002

Bertolín-Guillén J. M. “Intersexuality, transsexuality and mental health”. Annals of Psychiatry \& Mental Health, 2020; vol. 8 (núm. 3), pp. 1156. https://www.jscimedcentral.com/Psychiatry/psychiatry-8-1156.pdf

Bertolín-Guillén, J. M. "Informed consent and mental health, mainly in Spain, EU”. International Journal of Psychiatry and Mental Health, 2020; vol. 2 (núm. 1), pp. 1-7. https://doi.org/10.36811/ ijpmh.2020.110007

Bertolín-Guillén, J. M. «Las especialidades facultativas del ámbito de la salud mental ante los tribunales de justicia». Gaceta Internacional de Ciencias Forenses, 2021. [Article in Press]

Bertolín-Guillén, J. M. Deontología y confidencialidad en psiquiatría y psicología clínica en España. Revista de Bioética y Derecho, 2021; [vol. 23] (núm. 52), pp. 173-83. https://mcusercontent.com/ 002f6ac099bba449dcb2f6b0b/files/7eb58563-38d2-92c4-650a-0f4317ff63bc/rbyd52_10_art_bertolin.pdf

Clemente, M., Padilla-Racero, D. and Espinosa, P. “The dark triad and the detection of parental judicial manipulators. Development of a judicial manipulation scale”. International Journal of Environmental Research, 2020; vol.17 (núm. 8), pp. 2843. http://dx.doi.org/10.3390/ijerph17082843

CoHen, N. L. AND ŠTulHofer, A. "Correlates of adolescent ambiguity in defining their experience of sexual victimization in two large-scale Croatian samples". Journal of Child \& Adolescent Trauma, 2018; vol. 12 (núm. 2), pp. 211-20. https://doi.org/10.1007/s40653-018-0227-7

Díaz-Bustamante-Ventisca, M., Llovet-Rodríguez, C. y Narros-González, M. J. «Sexualización percibida en los estilismos de moda de niñas: un análisis transcultural en España-China». Comunicar, 2020; vol. XXVIII (núm. 65), pp. 77-87. https://doi.org/10.3916/C65-2020-07 
Engel, J., Kessler, A., Veit, M., Sinke, C., Heitland, I., Kneer, J. et Al. "Hypersexual behavior in a large online sample: Individual characteristics and signs of coercive sexual behavior". Journal of Behavioral Addictions, 2019; vol. 8 (núm. 2), pp. 213-22. https://doi.org/10.1556/2006.8.2019.16

García-Acero, M., Moreno-Niño, O., Suárez-Obando, F., Molina, M., Manotas, M. C., Prieto, J. C. ET AL. "Disorders of sex development: Genetic characterization of a patient cohort". Molecular Medicine Reports, 2020; vol. 21 (núm.1), pp. 97-106. https://doi.org/10.3892/mmr.2019.10819

GaRCíA-FernáNDEZ, M. A. «Delitos sexuales contra menores: especial referencia a agresiones y abusos sexuales». Revista Internacional de Doctrina y Jurisprudencia, 2020; vol. 23, pp. 15-43. http://ojs. ual.es/ojs/index.php/RIDJ/article/view/4327/4248

Gómez-Balaguer, M., Hurtado-Murillo, F., Modrego-Pardo, I. y Morillas-Ariño, C. «Identidad de género e identidad sexual: a veces coincidentes, pero no siempre lo mismo». Revista Desexología, 2020; vol. 9 (núm. 2), pp. 5-7. https://www.desexologia.com/wp-content/uploads/2020/12/Volumen-92-2020-diciembre.pdf

Hines, M. "Neuroscience and sex/gender: Looking back and forward". Journal of Neuroscience, 2020; vol. 40 (núm. 1), pp. 37-43. https://doi.org/10.1523/JNEUROSCI.0750-19.2019

Hruz, P. W. "Deficiencies in scientific evidence for medical management of gender dysphoria". The Linacre Quarterly, 2020; vol. 87 (núm. 1), pp. 34-42. https://doi.org/10.1177/0024363919873762

Hurtado-Murillo, F. y Pérez-Conchillo, M., Coord. Educación para la sexualidad con bases científcas. Documento de consenso de Madrid. Recomendaciones de un grupo internacional de expertos. Valencia, ES: Asociación Española de Especialistas en Sexología; 2011.

KALTIALA-HEINO, R., BERGMAN, H., TYÖLÄJÄRVI, M. and FRISÉN, L. "Gender dysphoria in adolescence: Current perspectives". Adolescent Health, Medicine and Therapeutics, 2018; vol 9, pp. 31-41. https://doi.org/10.2147/AHMT.S135432

Koops, T., Turner, D., Neutze, J. AND Briken, P. “Child sex tourism - prevalence of and risk factors for its use in a German community sample”. BMC Public Health, 2017; vol. 17 (núm. 1), pp. 344. https:// doi.org/10.1186/s12889-017-4270-3

Kvitкo, L. A. «Errores periciales y falsas denuncias de abuso sexual infantil». Revista Española de Medicina Legal, 2006; vol. 6, pp. 39-42. https://revistas.ucm.es/index.php/REML/article/ view/50198/46651

Lin, W. H., Liu, C. H. AND YI, C. C. "Exposure to sexually explicit media in early adolescence is related to risky sexual behavior in emerging adulthood". PLoS One, 2020; vol. 15 (núm. 4), pp. e0230242. https://doi.org/10.1371/journal.pone.0230242

LÓPEZ-CONTRERAS, R. E. «Interés superior de los niños y niñas: definición y contenido». Revista Latinoamericana de Ciencias Sociales, Niñez y Juventud, 2015; vol. 13 (núm. 1), pp. 51-70. http://www. scielo.org.co/pdf/rlcs/v13n1/v13n1a02.pdf

LuX, A., Kropf, S., Kleinemeier, E., Jürgensen, M., Thye, U. and The Dsd Network Working Group. "Clinical evaluation study of the German network of disorders of sex development (DSD)/intersexuality: Study design, description of the study population, and data quality". BMC Public Health, 2009; vol. 9, pp. 110. https://doi.org/10.1186/1471-2458-9-110

MAIDA, A. M., HERSKOVIC, V. y PRADO, B. «Síndrome de alienación parental». Revista Chilena de Pediatría, 2011; vol. 82 (núm. 6), pp. 485-92. http://dx.doi.org/10.4067/S0370-41062011000600002

Marote-González, R. M., Pera-Bajo, F. J., Perea-Pérez, B. y SÁnchez-SÁnchez, J. A. «Estudio de la transexualidad desde la Clínica Médico Forense de Madrid». Revista Española de Medicina Legal, 2018; vol. 44 (núm. 4), pp. 150-7. https://doi.org/10.1016/j.reml.2018.04.002

Moon, D. S., Lee, M. H., ChUng, D. S. And Kwack, Y. S. "Custody evaluation in high-conflict situations focused on domestic violence and parental alienation syndrome". Soa Chongsonyon Chongsin Uihak, 2020; vol. 31 (núm. 2), pp. 66-73. http://dx.doi.org/10.5765/jkacap.200004

Morant-Luján, Y. «Intersexualidad. ¿Qué es normal?» Revista Desexología, 2020; vol. 9 (núm. 1), pp. 4-6. https://www.desexologia.com/wp-content/uploads/2020/07/Volumen-91-2020-junio.pdf 
Moreno, C., Ramos, P., Rivera, F., SÁnchez-Queija, I., Jiménez-Iglesias, A., García-Moya, I. et AL. Resultados del estudio HBSC 2018 en España sobre conducta sexual. Análisis de tendencias 20022006-2010-2014-2018. Madrid: Ministerio de Sanidad; 2020. https://cpage.mpr.gob.es/

MurPhy, T. F. “Adolescents and body modification for gender identity expression”. Medical Law Review, 2019; vol. 27 (núm. 4), pp. 623-39. https://doi.org/10.1093/medlaw/fwz006

Nguyen, H. B., Loughead, J., Lipner, E., Hantsoo, L., Kornfield, S. L. And EpPerson, N. “What has sex got to do with it? The role of hormones in the transgender brain”. Neuropsychopharmacology, 2019; vol. 44 (núm. 1), pp. 22-37. https://doi.org/10.1038/s41386-018-0140-7

Patterson, J. G., Jabson, J. M. and Bowen, D. J. "Measuring sexual and gender minority populations in health surveillance". LGBT Health, 2017; vol. 4 (núm. 2), pp. 82-105. https://doi.org/10.1089/ lgbt.2016.0026

Pena, R., Lauar, H. And Barros, A. "Forensic psychiatry assessment during parental alienation claims: Two cases with different outcomes". Brazilian Journal of Psychiatry, 2020; vol. 42 (núm. 4), pp. 453-4. https://doi.org/10.1590/1516-4446-2020-0758

Phoenix, C. H., Goy, R. W., Gerall, A. A. And Young, W. C. "Organizing action of prenatally administered testosterone propionate on the tissues mediating mating behavior in the female guinea pig". Endocrinology, 1959; vol. 65 (núm. 3), pp. 369-82. https://doi.org/10.1210/endo-65-3-369

Rizvi, M. B., Conners, G. P. AND RABINER, J. "New York State child abuse, maltreatment, and neglect". In: StatPearls [Internet]. Treasure Island (FL): StatPearls Publishing; 2021. https://pubmed.ncbi. nlm.nih.gov/33351402/

Robles-García, R. y Ayuso-Mateos, J. L. «CIE-11 y la despatologización de la condición transgénero» [Editorial]. Revista de Psiquiatría y Salud Mental (Barc.), 2019; vol. 12 (núm. 2), pp. 65-7. https://www.elsevier.es/es-revista-revista-psiquiatria-salud-mental--286-pdf-S1888989119300035

Rodríguez-Molinero, L. «La adolescencia y los riesgos sexuales» [Editorial]. Adolescere, 2019; vol. 7 (núm. 1), pp. 3-4. https://www.adolescenciasema.org/ficheros/REVISTA\%20ADOLESCERE/ vol7num1-2019/3-4\%20Editorial\%20-\%20La\%20adolescencia\%20y\%20los\%20riesgos\%20sexuales.pdf

SAncho-Conde, T. Minoría de edad y delincuencia sexual: consecuencias jurídicas aplicables a menores que cometen delitos contra la libertad e indemnidad sexual. [Tesis doctoral]. Tarragona: Departamento de Derecho Público, Universitat Rovira i Virgili; 2020. https:/www.tesisenred.net/bitstream/ handle/10803/669895/TESI\%20TATIANA\%20SANCHO.pdf?sequence=1\&isAllowed=y

TORRES-FERnÁNDEZ, M. E. «Abusos sexuales sobre personas incapacitadas para oponerse a su agresor y sobre incapaces para consentir». Revista Española de Medicina Legal, 2020; vol. 46 (núm. 4), pp. 198-9. https://doi.org/10.1016/j.reml.2020.03.001

Verrocchio, M. C., Marchetti, D., Carrozzino, D., Compare, A. And Fulcheri, M. "Depression and quality of life in adults perceiving exposure to parental alienation behaviors". Health and Quality of Life Outcomes, 2019; vol. 17 (núm. 1), pp. 14. http://dx.doi.org/10.1186/s12955-019-1080-6

VILALTA, R. y Nodal, M. W. «Sobre el mito del síndrome de alienación parental (SAP) y el DSM-5». Papeles del Psicólogo, 2017; vol. 38 (núm. 3), pp. 224-31. https://doi.org/10.23923/pap.psicol2017.2843

VILJOEN, B. L. "Unintentional state enforced parental alienation syndrome during the hard lockdown in South Africa”. The Family Journal, 2021. [OnlineFirst] https://doi.org/10.1177/1066480720987998

Xu, Y., Norton, S. And Rahman, Q. “Adolescent sexual behavior patterns in a British birth cohort: A latent class analysis”. Archives of Sexual Behavior, 2021; vol. 50 (núm. 1), pp. 161-80. https://doi. org/10.1007/s10508-019-01578-w 